\title{
Design of Lead Spectrometer for Spent Fuel Fissile
}

\section{Assay}

\author{
Yong Deok Lee, Chang Je Park, Ho-Dong Kim and Kee Chan Song \\ Nonproliferation System Research Department, Korea Atomic Energy Research Institute, Daejeon 305-353, Korea
}

Received: June 04, 2013 / Accepted: August 12, 2013 / Published: January 31, 2014.

\begin{abstract}
The design of a lead slowing down spectrometer is being developed at KAERI (Korea Atomic Energy Research Institute) for an analysis of isotopic fissile content in spent and recycled fuel. The lead spectrometer has an advantage to assay fissile content directly from spent fuel. Accurate fissile material data must be fed back to the spent fuel design and management. For reutilization of PWR spent fuel, an isotopic fissile content analysis is required to obtain safety and economics in the fuel cycle. The LSDS (lead slowing down spectrometer) uses the different fission signatures of each fissile with respect to the interrogation neutron energy. Several simulations were done and parameter was obtained for the system working. Energy between $1 \mathrm{keV}$ to $0.1 \mathrm{eV}$ is very sensitive to isotopic fissile material fission with good energy resolution. An external source neutron is necessary to induce enough fissile fission. A neutron production methodology is being developed for cost effectiveness, easy maintenance and high neutron yield rate. The threshold fission detector screens the prompt fast fission neutrons from an intense radiation background. The detection of a fission signature has a direct relationship with the isotopic fissile content.
\end{abstract}

Key words: Fissile assay, lead slowing down spectrometer, fission measurement, fissile utilization, neutron source, resolution.

\section{Introduction}

An assay technology for isotopic fissile material contents in spent and recycled fuel is being developed at KAERI (Korea Atomic Energy Research Institute) using a LSDS (lead slowing down spectrometer) [1]. The new technology has several features for isotopic fissile content analysis: a direct isotopic fissile assay (not a total fissile assay), no interference from the intense radiation background by the spent fuel or recycled material (mainly; by spontaneous fission neutrons) and no requirement of the burnup history.

The accumulation of spent fuel is also a big issue in Korea because of a limited storage capacity. The amount of spent fuel will reach maximum capacity soon. To reuse the fissile materials in PWR spent fuel, in a sustainable manner of national energy supply, a future nuclear energy system is under development, an

Corresponding author: Yong Deok Lee, researcher, research fields: nuclear fissile assay, radiation measurement, fuel cycle and nondestructive assay. E-mail: ydlee@kaeri.re.kr.
SFR (sodium fast reactor) linked with the pyrochemical process. The pyroprocess uses PWR spent fuel and produces a source material to fabricate the fuel rod of an SFR. Therefore, an isotopic fissile content assay is very important for reuse of PWR spent fuel in an SFR and optimum storage design as well. The fissile content must be fed back to its storage design and the accuracy of the fissile content must be verified in the reuse of PWR spent fuel for fuel safety and economics.

The LSDS uses a different fission signature of each fissile with respect to the interrogation neutron energy $[2,3]$. An external source neutron is produced by a $(e$, $\gamma)(\gamma, n)$ reaction and the neutron slows down its energy continuously by an interaction with a lead medium. Therefore, a lead slowing down spectrometer is very sensitive to produce isotopic fissile fission. The spectrometer design was performed based on 16 by 16 spent fuel assembly and 2 by 2 pyro assembly. The distance between the fuel assay area and source 
geometry was determined by considering the source neutron intensity and energy spectrum. A threshold fission detector screens the prompt fast fission neutrons from intense radiation background. The self shielding property was simulated for PWR spent fuel and pyro material [4]. The fission characteristics for pyro and spent fuel were investigated in the spectrometer. The parameter between energy and time was obtained. The source neutron production mechanism was decided using an accelerator and the target was designed to produce neutrons effectively. The neutron production rate was examined at the target.

Another important application of LSDS is to verify the theoretical burnup and provide a correction factor for improving the fissile material content, fission product content and theoretical burnup. Additionally, an accurate isotopic fissile content assay will increase the transparency and credibility for spent fuel management and the reuse of PWR spent fuel, with an international increase in demand.

\section{Lead Slowing down Spectrometer}

The lead spectrometer consists of several parts: a lead slowing down medium, an external neutron source, a threshold fission detector, a data acquisition and analysis $\mathrm{s} / \mathrm{w}$, as shown in Fig. 1. An intense neutron generator is required to obtain the enough fission signals from the fissionable materials (uranium and plutonium isotopes) and to receive a good detection statistics without background interference. As a source neutron, $\sim 10^{12} \mathrm{n}$ 's/s were decided using one section electron linear accelerator. The neutrons are produced in a tantalum or tungsten target material which has multiple layers with a different thickness to maximize the neutron production [5]. The gap between layers was determined for a path of coolant.

The spent fuel assembly, a $16 \times 16$ fuel type with a 4.5 initial enrichment and 50,000 MWD/MTU, were referenced [6]. $\sim 1 \mathrm{wt} \%$ of $\mathrm{U} 235, \sim 0.6 \mathrm{wt} \%$ of Pu239, $\sim 0.1 \mathrm{wt} \%$ of Pu241 and $95 \mathrm{wt} \%$ of U238 were used in the simulation. For the pyro material, $0.045 \mathrm{wt} \%$ of

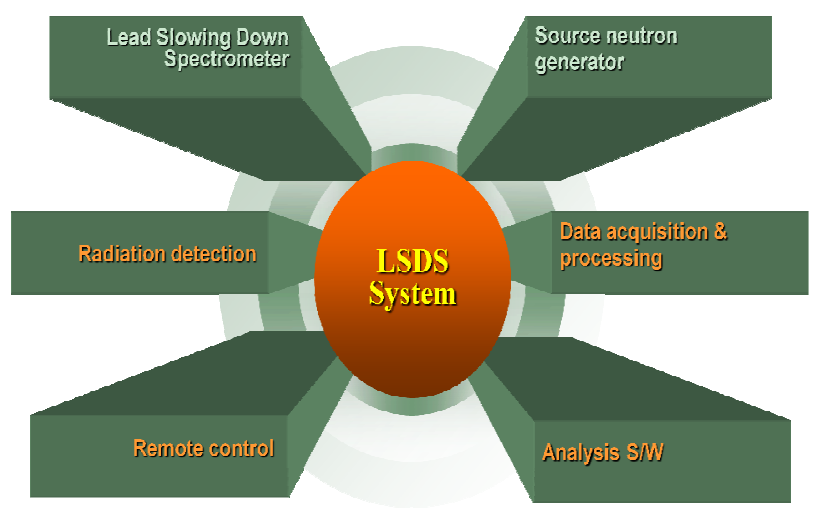

Fig. 1 LSDS system component.

U235, 11wt $\%$ of Pu239, 8.1wt $\%$ of Pu240, 1.69wt $\%$ of Pu241 and $64.64 w t \%$ of U238 were used in the simulation and a $2 \times 2$ assembly was chosen. The fuel assay area is located $40 \mathrm{~cm}$ from the neutron source at the center of the lead. The source neutron, having $\sim 0.5$ $\mathrm{MeV}$ mean energy, slows down continuously by inelastic and elastic scattering in the medium and induces fissile fission with respect to the slowing down energy [7]. The fission by fissile isotopes with respect to neutron energy is detected at the surrounding threshold fission chamber. The threshold detectors are a good choice for selecting only prompt fast fission neutrons from the fissile in a complex radiation field; neutron background, intense gamma rays and interrogated neutrons.

In the fissile content assay of spent fuel, the difficulty is the intense emission of neutrons by curium isotopes. The content of curium is relatively very small, but, the neutron emission is more than one hundred times greater than that by other nuclear materials. Fig. 2 shows the nuclear material production and neutron emission rate [6]. Therefore, an indirect way was simply used to obtain fissile content through the help of a burnup code. In the simple verification of spent fuel or nuclear materials, curium measurement with a burnup code has an advantage to extract the fissile content.

\subsection{LSDS System Property}

A simulation on the fuel assay area was done in order to examine the fission property change by the 


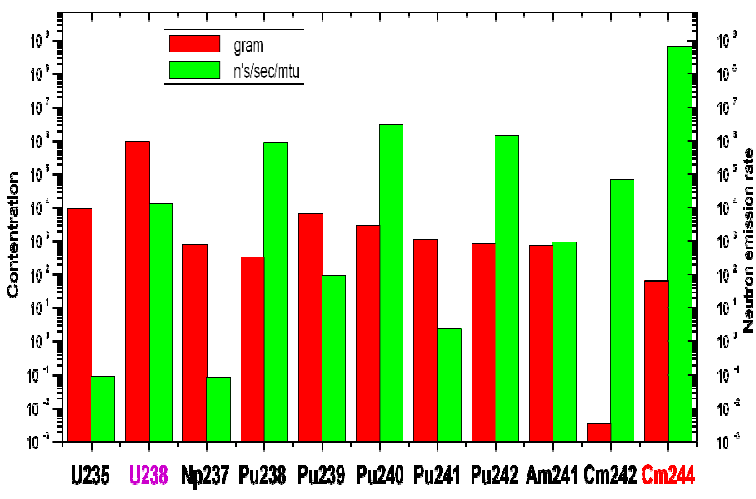

Fig. 2 Production of nuclear materials and neutron emission rate $(4.5 w t \%$ initial enrichment and 50 GWd/MTU burnup).

fissile content change. The amount of Pu239 is dominant in the pyro material. U235 is relatively small amount comparing to that of Pu239 and Pu241. Fig. 3 shows the normalized fission rate for pyro material, 11 wt\% of Pu239, at the slowing down time by changing assembly type and rod length. In the fuel area, the fission characteristics of the fissile is expressed as:

$$
\emptyset_{\text {rod }}^{\text {fission }}=\int_{\mathrm{t}_{1}}^{\mathrm{t}_{2}} \int_{0}^{\infty} \int_{\text {rod }} v(E) \phi(r, E, t) \mathrm{d} r \mathrm{~d} E \mathrm{~d} t
$$

where, $v(\mathrm{E})$ is the fission rate and $\phi(r, \mathrm{E}, t)$ is the source neutron arriving at the fuel area.

In Fig. 3, the results show a shift by the increase in the number of rods. Moreover, between $200 \mu$ s and 500 $\mu \mathrm{s}$, a large number of rods provide a greater decrease than a few rods. The results can show the limitation of the fuel rod assay. However, in changing the rod length with the same fuel area, the result does not show much difference in the fission signature over the entire slowing down time. Therefore, from the results, the total number of rods is more sensitive in fission change than the length.

In fission property, $\mathrm{U} 235$ has a big resonance around $1 \mathrm{eV}$ and $0.3 \mathrm{eV}, 10 \mathrm{eV}$ and $70 \mathrm{eV}$ for Pu239 [3]. U238 is very large amount in the assembly as a base material, but the fission contribution is very small. Fig. 4 shows the fission signature by fissile materials in a $16 \times 16$ spent fuel assembly. For the assembly, the fission signature of U235, Pu239 and Pu241 is more dominant than that of Pu240 and U238. Specially, between $20 \mu \mathrm{s}$

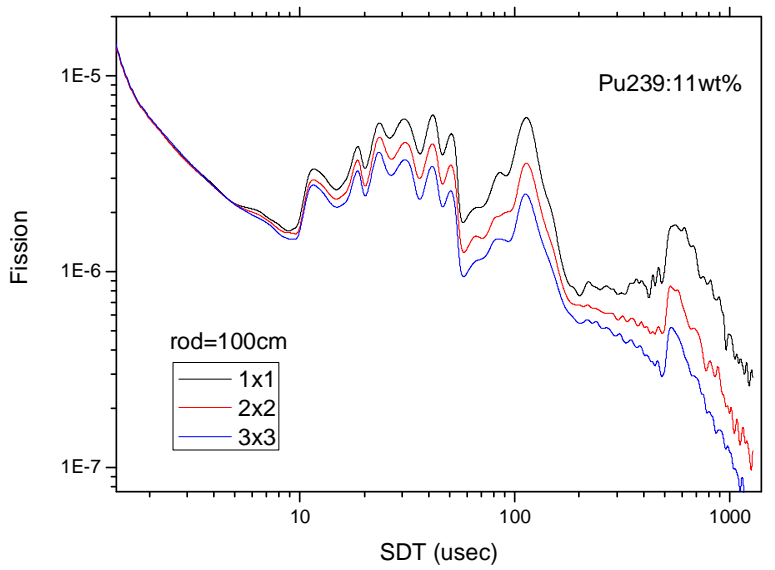

(a) Different cross section area

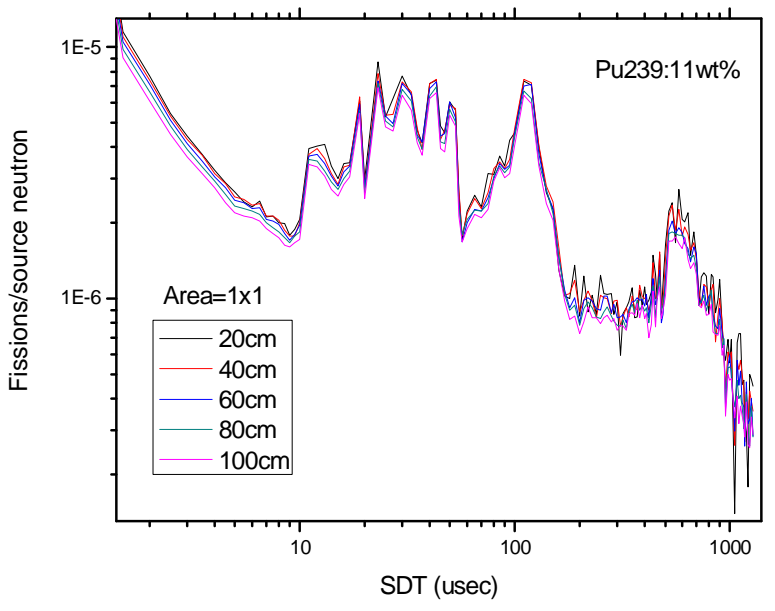

(b) Different rod length

Fig. 3 Normalized fission characteristics by different fuel area for pyro material.

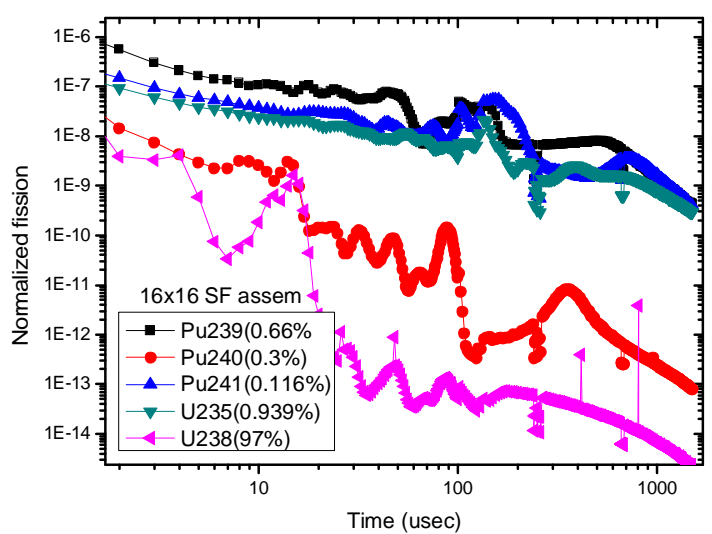

Fig. 4 Fission characteristics of fissile (U235, Pu239, Pu241) by interrogation source neutrons $(16 \times 16$ spent fuel assembly, 4.5\%, $50 \mathrm{Gwd} / \mathrm{MTU})$.

and $700 \mu$ s, each fissile has its fission structures. Therefore, this range which represents the fission characteristics can be used for the isotopic fissile assay. 
The fission signature has a direct relationship with the isotopic fissile content.

In lead geometry, the energy and time relationship is expressed as below:

$$
E=k /\left(t+t_{0}\right)^{2}
$$

Generally, $k$ value is expressed as a function of the geometry property and $t$ represents the slowing down time. Table 1 shows the results of energy and time relation in lead medium. The energy range from $0.1 \mathrm{eV}$ to $1 \mathrm{keV}$ was referenced.

Usually, the fuel rod length is much longer than the detector size. Therefore, a scanning mechanism is required to get the axial fissile distribution. Fig. 5 shows 10 fuel sub-cells in a rod and the detector size. A 1 inch detector diameter and the $20 \mathrm{~cm}$ length were selected in the calculation. The detection sensitivity from fissile fission neutron is expressed as below:

$$
\int_{\mathrm{S}_{\mathrm{det}}} \int_{0.1 \mathrm{MeV}}^{\infty} \sigma_{f} \phi_{f}(r \cdot E, t) \mathrm{d} E \mathrm{~d} A
$$

where, $\sigma_{f}$ is the detector fission X-section at the detector and $\phi_{f}$ is the fissile fission neutron arriving at the detector. The result from sub-cells was summarized in Fig. 5. Sub-cell numbers 4-7 are main contribution to the detection. Therefore, scanning can cover the entire fuel length and it depends on the detector length.

\subsection{Source Neutron System}

A neutron source is required to induce fissile fission in LSDS. A linear accelerator has an advantage to increase its energy and current simply, when necessary. Fig. 6 shows a schematic view of the electron linear accelerator system. Electrons are generated in e-gun and focused through buncher. The focused beam is accelerated in accelerating column until several tens of $\mathrm{MeV}$. The accelerated beam arrives at target through guiding tube. Electron beam finally hits the target which has multiple tantalum layers. By an $(e, \gamma)(\gamma, n)$ reaction, evaporation neutrons are produced which has an average energy, $\sim 0.5 \mathrm{MeV}$.

Several options were considered in the electron accelerator setup to produce neutrons effectively and efficiently. An electron gun was manufactured as a first
Table 1 Parameters for relationship between energy and time in lead medium.

\begin{tabular}{lllll}
\hline \multirow{2}{*}{$\begin{array}{l}\text { Energy } \\
\text { range }\end{array}$} & \multicolumn{4}{c}{ Lead medium } \\
\cline { 2 - 5 } $0\left(\mathrm{eV}-\mu \mathrm{s}^{2}\right)$ & error & $t_{o}(\mu \mathrm{s})$ & error \\
\hline $0.1-1 \mathrm{eV}$ & $167,033.789$ & $1,525.69$ & 0.6764 & 0.067 \\
\hline
\end{tabular}

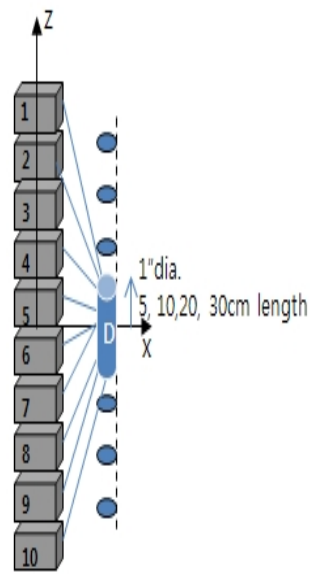

(a) Fuel(sub-cells) and detector configuration

\begin{tabular}{ccc}
\hline Sub-cell No. & Detection $(>0.1 \mathrm{MeV})$ & Relativity $(\%)$ \\
\hline 1 & $9.24916 \mathrm{E}-6$ & 1.5 \\
2 & $1.92257 \mathrm{E}-5$ & 3.2 \\
3 & $5.81046 \mathrm{E}-5$ & 9.6 \\
4 & $3.05715 \mathrm{E}-4$ & 51.0 \\
5 & $5.98135 \mathrm{E}-4$ & 100 \\
6 & $5.98135 \mathrm{E}-4$ & 100 \\
7 & $3.05074 \mathrm{E}-4$ & 51.0 \\
8 & $5.78302 \mathrm{E}-5$ & 9.6 \\
9 & $2.21295 \mathrm{E}-5$ & 3.6 \\
10 & $9.79040 \mathrm{E}-6$ & 1.6 \\
\hline
\end{tabular}

(b) Detection from sub-cells

Fig. 5 Geometry of $\mathbf{1 0}$ sub-cells in a fuel rod and detection along axial direction (1 inch diameter and $20 \mathrm{~cm}$ long detector).

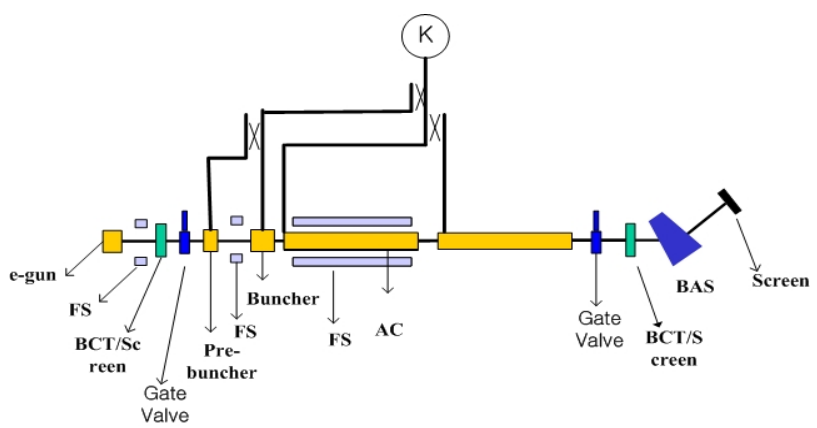

Fig. 6 Schematic view of electron linac to produce source neutron.

step and the performance test was done. The electron current after an e-gun at the BCM (beam current monitor) was measured. 1.4 A were obtained at e-gun accelerating high voltage, $80 \mathrm{kV}$. Table 2 summarizes 
the measured beam current at different high voltage and pulses.

To design the target, the neutron production simulation [8] was done with different electron energies. Fig. 7 shows the neutron production rate with different electron energies. The production has a maximum value between $0.5 \mathrm{~cm}$ and $1 \mathrm{~cm}$ in thickness, even though the target material and incident electron energies are different. From the simulation, at $35 \mathrm{MeV}$ of electron energy, about 150 electrons are required to produce one neutron per unit time [8]. Fig. 8 shows the neutron production rate at a target with different electron pulse widths. Neutron generation is expected to be influenced by the pulse width. The short pulse shows the high neutron production rate in the simulation.

Fig. 9 shows the designed target geometry. The geometry was based on the beam diversion property when the beam passes through the layers. The target consists of five layers with different thickness. Gap is positioned in between layers to pass coolant. 30-40 $\mathrm{MeV}$ of electron energy is considered with a 240 repetition rate. About $500 \mathrm{~mA}$ current will be considered at the target.

\section{LSDS System}

From the simulation and calculations, the LSDS system was setup as shown in Fig. 10. The target is located at the center of a lead cube. The threshold fission chamber detects the prompt fast fission neutrons. In the LSDS, a broad range of interrogation neutron energies is available in the lead. Energy between $1 \mathrm{keV}$ to $0.1 \mathrm{eV}$ is very sensitive to the fissile material fission and a different fission signature of the fissile isotopes allows us to distinguish these isotopes from each other. The lead also serves as a shield to the intense gamma rays from spent fuels and target. LSDS system is very appropriate tool to get isotopic fissile content.

\section{Results and Discussion}

The material composition of the pyro is much different from the spent fuel. The concentration of
Pu239 is much higher than that of spent fuel. From the simulation, each fissile has its own fission characteristics during the neutron slowing down. After a $20 \mu$ s slowing down time, each fissile shows its fission property to identify from others. However, after

Table 2 Electron current of e-gun system at beam current monitor.

\begin{tabular}{lllll}
\hline $\begin{array}{llll}\text { Bias } \\
(\mathrm{V})\end{array}$ & $\begin{array}{l}\text { H.V. } \\
(\mathrm{kV})\end{array}$ & $\begin{array}{lll}\text { Pulser } & \text { Width (ns) } & \text { Voltage }(\mathrm{V}) \\
(\mathrm{A})\end{array}$ \\
\hline 100 & 40 & 100 & 300 & 0.4 \\
100 & 50 & 100 & 300 & 0.6 \\
100 & 60 & 100 & 300 & 0.8 \\
100 & 70 & 100 & 300 & 1.1 \\
100 & 80 & 100 & 300 & 1.4 \\
\hline
\end{tabular}

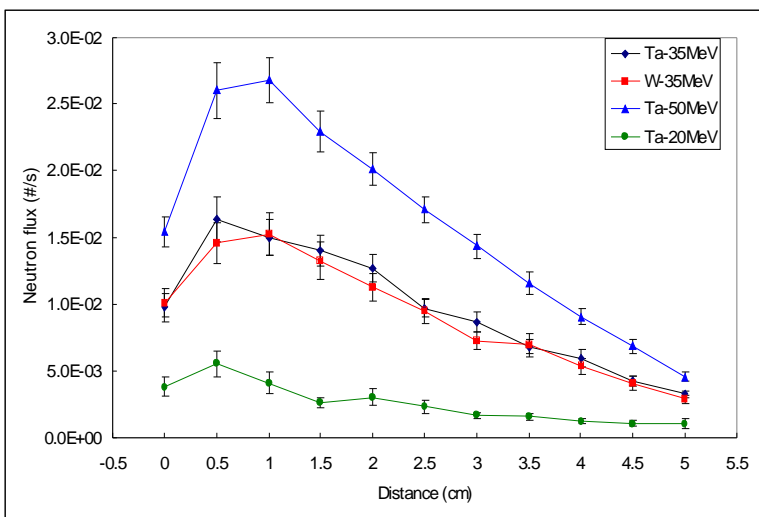

Fig. 7 Neutron production rate at different electron energies.

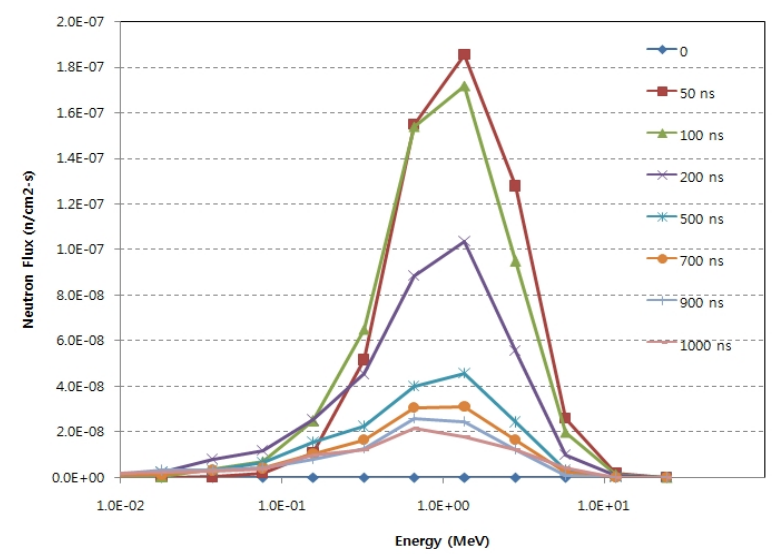

Fig. 8 Neutron production rate with different electron pulse width.

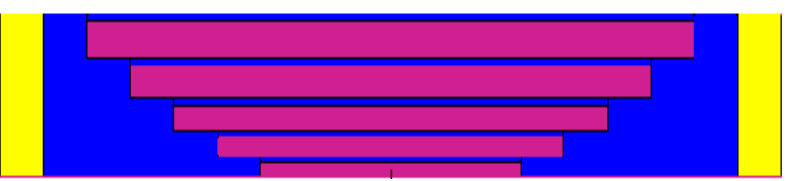

Fig. 9 Target geometry to produce neutron. 


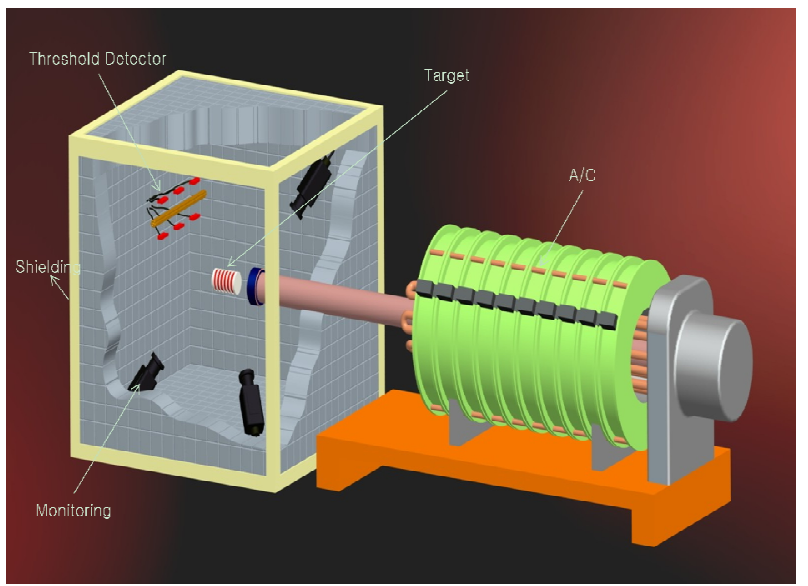

Fig. 10 Schematic view of LSDS system.

$800 \mu \mathrm{s}$, there are no dominant fission structures to distinguish it from the others. Specially, the fission characteristic variation depends more on the number of rods than the rod length. Scanning method can cover a whole assembly length. The threshold fission chamber detector is a good choice for resolving the fission characteristics to distinguish the fission neutrons from the background.

The neutron production at the target using electron acceleration was decided. From the results on the neutron production, target geometry was setup and acceleration parameters were decided to get effective and efficient neutron production. As a first step, the electron current was measured. The maximum current measurement will be performed to produce high neutron yield. However, equipment setup including acceleration is necessary in the future.

\section{Conclusions}

The LSDS has good features for a direct analysis of the content of isotopic fissile material in spent fuel and reuse of spent fuel. The dominant feature of LSDS is not to be interfered from the intense radiation background. However, to overcome the background, an intense neutron source is required. From a simulation and calculation, the total LSDS system was designed conceptually. However, the actual measurement after the system setup is required to approve the capability of an isotopic fissile content assay.

The LSDS system is applicable to the fuel cycle and spent fuel management as well. An accurate isotopic fissile assay will contribute to an increase in transparency of spent fuel management and safety for the reuse of PWR spent fuel. In addition, an advanced isotopic fissile assay will contribute to international safeguards technology development.

\section{Acknowledgments}

This work was supported by the Nuclear Research Foundation of Korea (NRF) grant funded by the Korea gevernment (MSIP) (No. 2013036069).

\section{References}

[1] Y.D. Lee, C.J. Park, G.I. Park, K.C. Song, Design of Lead Slowing Down Spectrometer for Spent Fuel Fissile Assay, INMM, Palm Desert, 2011.

[2] A. Gavron, Spent Fuel Assay with a Lead Slowing down Spectrometer, LANL, 2010.

[3] Y. Danon, Experiments with a Lead Slowing-down Spectrometer for Assay of Used Nuclear Fuel, Rensselaer Polytechnic Institute, 2012.

[4] Y.D. Lee, C.J. Park, G.I. Park, K.C. Song, Self shielding in nuclear fissile assay using LSDS, in: 2012 Spring Meeting of the KNS, Jeju, May 16-18, 2012, pp. 345-346.

[5] C.J. Park, M.K. Jaradat, L. Alawneh, Y.D. Lee, Metal plate target design for the lead slowing down time spectrometer (LSDTS), Annals of Nuclear Energy 49 (2012) 218-222.

[6] A.G. Croff, ORIGEN2: Isotope Generation and Depletion Code Matrix Exponential Method, Oak Ridge National Laboratory, 1985.

[7] K. Anderson, Update on Establishing the Feasibility of Lead Slowing Down Spectrometer for Direct Measurement of Plutonium in Used Fuel, INMM, Orlando, 2012.

[8] D.B. Pelowitz, MCNP: A General Monte Carlo Code for Neutron and Photon Transport, LA-CP-05-0369, Los Alamos National Laboratory, 2005. 